\title{
High-resolution accelerator alignment using x-ray optics
}

\author{
Bingxin Yang and Horst Friedsam \\ Advanced Photon Source, Argonne National Laboratory, Argonne, Illinois 60439, USA
}

(Received 21 December 2005; published 3 March 2006)

\begin{abstract}
We propose a novel alignment technique utilizing the x-ray beam of an undulator in conjunction with pinholes and position-sensitive detectors for positioning components of the accelerator, undulator, and beam line in an x-ray free-electron laser. Two retractable pinholes at each end of the undulator define a stable and reproducible $\mathrm{x}$-ray beam axis (XBA). Targets are precisely positioned on the XBA using a pinhole camera technique. Position-sensitive detectors responding to both $\mathrm{x}$-ray and electron beams enable direct transfer of the position setting from the XBA to the electron beam. This system has the potential to deliver superior alignment accuracy $(1-3 \mu \mathrm{m})$ for target pinholes in the transverse directions over a long distance ( $200 \mathrm{~m}$ or longer). It can be used to define the beam axis of the electron-beam-based alignment, enabling high reproducibility of the latter. This x-ray-based concept should complement the electron-beam-based alignment and the existing survey methods to raise the alignment accuracy of long accelerators to an unprecedented level. Further improvement of the transverse accuracy using x-ray zone plates will be discussed. We also propose a concurrent measurement scheme during accelerator operation to allow real-time feedback for transverse position correction.
\end{abstract}

DOI: 10.1103/PhysRevSTAB.9.030701

PACS numbers: 41.60.Cr, 29.17.+w, 41.50.+h, 41.75.Lx

\section{INTRODUCTION}

Several large-scale linear accelerator projects are under construction or have been proposed. The Linear Coherent Light Source (LCLS) utilizes parts of the existing linear accelerator at SLAC followed by a 130-m-long undulator section with a $6.8-\mathrm{mm}$ fixed gap [1]. DESY is planning to build an $\mathrm{x}$-ray free-electron laser (XFEL) with a total length on the order of $3.3 \mathrm{~km}$ for multiple undulator sections of which the longest is about $300 \mathrm{~m}$ [2]. The gap in this device varies from 6 to $20 \mathrm{~mm}$ depending on the undulator type utilized in each of the beam lines. A proposal by SPring- 8 to establish the SCSS will use a $1-\mathrm{GeV}$, 55-m-long accelerator followed by a 22.5 -m-long invacuum undulator section with a 3.7-mm gap [3]. Last, but not least, PAL at POSTECH in South Korea is proposing the PAL/XFEL with a 60-m in-vacuum variable-gap undulator with a minimum gap of $3 \mathrm{~mm}$ [4]. Finally, the International Linear Collider (ILC) project now being developed in a global design effort will need highprecision alignment of accelerating and diagnostics components over distances of 10-20 km [5,6].

All of these projects require precision alignment of beam line components at unprecedented accuracy, including the vacuum chambers. In the LCLS, for example, many undulator components need to be aligned to better than $20 \mu \mathrm{m}$ from a straight line designated to be the beam axis.

Using modern survey technology one can establish a control reference system with point accuracies of \pm 150 to $\pm 300 \mu \mathrm{m}$ depending on the size of the object. In long stretched objects such as linear accelerators or freeelectron lasers (FELs), large-scale deviations due to the accumulation of small remaining systematic instrument errors and environmental effects can even exceed these tolerances and directly affect the positioning of the
XFEL components. State-of-the art hydrostatic level sensors deliver an accuracy of $\pm 5 \mu \mathrm{m}$ in the vertical direction and wire position sensors (WPSs) are under development providing an accuracy of $\pm 1.5 \mu \mathrm{m}$ only in the horizontal plane due to uncertainties in the wire sag. With these instruments it is possible to achieve relative alignment tolerances on the order of $\pm 25 \mu \mathrm{m}$ or better when considering transfer errors from the monitors to the beam components [7]. However, the accuracy of these devices is limited by the length of the object to be measured and on-axis alignment is not possible. Minimizing the transfer error becomes even more difficult since two separate systems are required to position and monitor vertical and horizontal deviations.

In 1968 Herrmannsfeldt and others proposed and implemented a precision straight-line alignment system utilizing Fresnel lenses for the 3-km-long Stanford linear accelerator [8]. The system uses a HeNe laser light source, a detector, and rectangular Fresnel lenses with distancedependent focal lengths to project the source onto the detector. The accelerator is supported by 12 -m-long linked girders with an integrated strongback for the accelerating structures and a $60 \mathrm{~cm}$-diameter light pipe for the alignment system. At each girder link a Fresnel lens can be inserted into the light beam creating a diffraction pattern at the detector. Deviations relative to an initial straight-line setup using a well-defined Fresnel target and the detector zero position can be measured. Moving the strongback support with the Fresnel lens to the zero position, any link can be placed on axis and with that the accelerating structure. The estimated transverse accuracy of this system is on the order of $\pm 25 \mu \mathrm{m}$ over the length of the accelerator, without considering the transfer error from the alignment system to the actual beam axes. 
A similar system was proposed in 1990 by Griffith and his colleagues for the construction of an FEL at Lawrence Livermore Laboratory $[9,10]$. This concept uses the Poisson spot created as a diffraction pattern by an opaque sphere substituting for a Fresnel lens to align the accelerator. It includes an elaborate feedback system to maintain the pointing direction of an expanded light beam and eliminates the need for inserting and removing reference targets as required in the SLAC design, as many spheres can occupy the cross section of the light beam. This system was designed to provide transverse positioning on the order of $\pm 25 \mu \mathrm{m}$ over a 300 -m-long FEL.

In order to overcome these limits in survey accuracy, the electron-beam-based alignment (EBBA) technique was developed in the last decade to align magnetic quadrupoles and sextupoles. When an electron-beam trajectory deviates from the centers of these magnets, it experiences a transverse kick. Since the strength of the kick depends on the strength of the magnets and the beam energy, the trajectory deviations in the magnets can be detected by monitoring the downstream beam position monitors (BPMs) while varying the strength of the magnet or electron energy. Through an iterative procedure of correcting magnet positioning errors and measuring the BPM signal, the EBBA technique can now attain micron-level accuracy under ideal conditions $[11,12]$. However, this technique is sensitive to the presence of a dipole field that cannot be turned off during the alignment process. Even the Earth's field can adversely affect the outcome. Finally, since no fixed monument is used to define the electron-beam axis (EBA), it is not predetermined to what line the final convergence will lead. Hence the EBA cannot be accurately reproduced after magnets are moved.

Similar to many previous authors, we also prefer the optical technique since photons travel in straight lines invacuum and in the magnetic field, with their trajectory curving less than $1 \mathrm{prad} / \mathrm{km}$ as a result of Earth's gravity. However the previous techniques have two major drawbacks. First, the diffraction of a visible light beam requires large apertures $R \gg \sqrt{\lambda S}$ over a long working distance $S$ [13], where $\lambda=\lambda / 2 \pi$ is given by the wavelength $\lambda$ of the light beam. This makes them incompatible with the small bore of modern accelerator chambers. Second, these techniques establish straight lines nearly a meter away from the main electron-beam axes, and the transfer error is not negligible.

Recently, Shintake et al. made a step towards overcoming these difficulties by proposing an on-axis alignment procedure using a laser beam and an Airy diffraction pattern for the SCSS x-ray FEL project [14]. They plan to enlarge the optical aperture by opening the gap of invacuum undulators during the alignment. An iris is attached to the geometric center of each cavity BPM. A laser beam is used to image the iris on to a CCD camera downstream to detect the transverse misalignment. The esti- mated transverse accuracy of positioning the geometric centers of the BPMs is on the order of several micrometers over the distance of 25 meters. Since most FELs do not have variable vacuum chambers, it will be difficult to apply this technique to other accelerators. Furthermore, it is not clear how much error will be induced during the crucial step of fiducializing the BPM and electronics when the difference between the geometric and electric centers of the BPM is measured, and when the fiducialization drifts due to temperature variations during the field installation.

In this work, we propose to use an x-ray beam, with a wavelength of $\lambda \sim 0.02-2 \mathrm{~nm}$, for on-axis alignment of pinhole apertures. We also propose to establish the $\mathrm{x}$-ray beam axis coincident with the main electron-beam axis by performing in situ fiducialization, which transfers the x-ray beam position to the electron beam using devices that interact with both $\mathrm{x}$-ray and electron beams, such as wire scanners or fluorescent screens. Since the diffraction effect is proportional to the square root of the wavelength, the alignment beam diameter can be kept small for x-ray photons, on the order of $1 \mathrm{~mm}$ over long distances $(>200 \mathrm{~m})$, and hence can be contained in an ordinary accelerator chamber. Another benefit is the vastly reduced scattering by gas molecules in the beam path since the scattering cross section is reduced by several orders of magnitude in the $x$-ray region. In order to reap these obvious benefits of using an x-ray beam for precision alignment, a proper procedure needs to be established.

In Sec. II, we start by describing a procedure utilizing a dedicated alignment undulator as an x-ray source and pinholes as alignment targets. We estimate the alignment accuracies using geometric and wave optics. In Sec. III, we discuss how to choose suitable $\mathrm{x}$-ray optical components, from the source to the detector for an x-ray alignment system. We discuss offline and online fiducialization issues in Sec. IV, improvements and other related issues in Sec. V, and summarize the paper in Sec. VI. Most of our discussion will be based on the geometry and parameters of the LCLS, but it can be easily extended to other large-scale accelerator projects. We envision that this technology could be beneficial for the ILC project with its component alignment tolerances even more challenging than what is currently required for the XFEL projects.

\section{X-RAY OPTICS ALIGNMENT PROCEDURE AND ACCURACY}

Figure 1 shows the schematics of the $\mathrm{x}$-ray instrument. An alignment undulator is located $20 \mathrm{~m}$ or more upstream of the accelerator beam line to be positioned with a retractable x-ray pinhole (source pinhole) located midway between the two. The pinhole is used to define a fixed source point for the alignment. An x-ray imaging detector is located $10 \mathrm{~m}$ or more downstream of the main beam line. A second retractable $\mathrm{x}$-ray pinhole (detector pinhole) is located immediately upstream of the detector, used to 


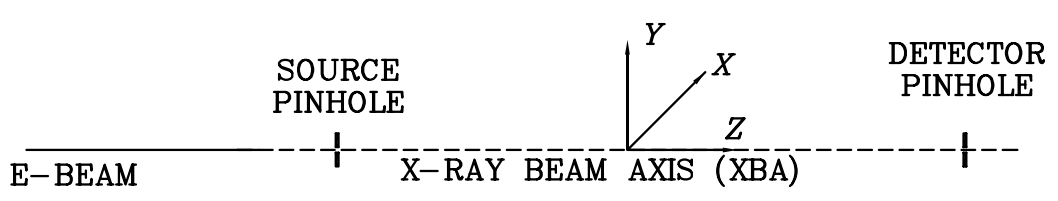

(a) Definition of coordinates

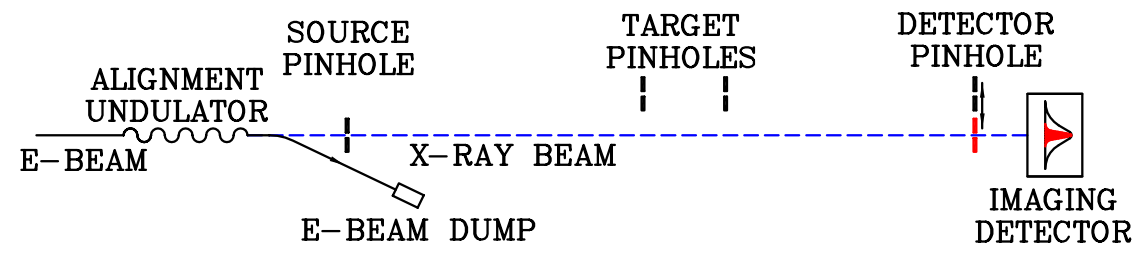

(b) Setup detector and undulator

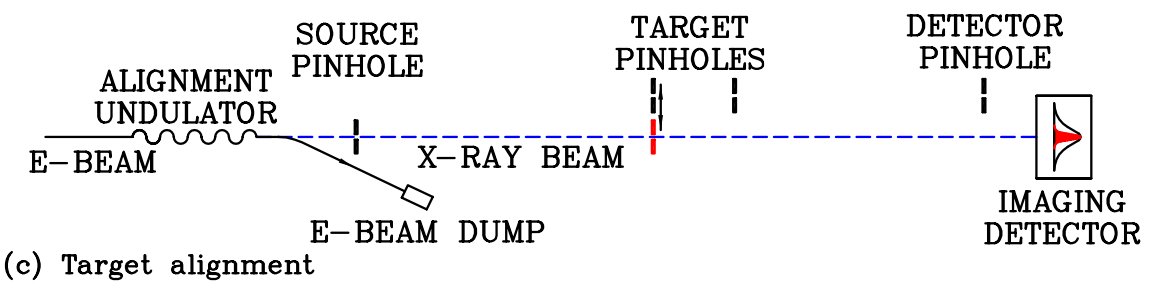

FIG. 1. (Color) Schematics showing the alignment procedure: (a) Definition of the XBA coordinate system, (b) setup of the XBA, and (c) alignment of target pinholes.

define the center of the detector plane. When both of the two $\mathrm{x}$-ray pinholes are inserted they define a straight line, which will be referred to as the $\mathrm{x}$-ray beam axis (XBA). In addition, every component to be aligned with the $\mathrm{X}$-ray beam will need a retractable pinhole (target pinhole) as an alignment target.

\section{A. Setup of the $x$-ray beam axis and primary coordinate system}

The reference XBA is established by conventional survey method, using common survey tools to set up the source and detector pinholes, both at inserted positions, onto the design electron-beam axis at the best achievable accuracy [Fig. 1(a)]. Once the two pinholes are set, the conventional survey monument network becomes secondary. The primary coordinates are now uniquely defined by the following rules: The $z$ axis or the XBA is the straight line linking the centers of the two pinholes, from the source to the detector. The $O y z$ plane is defined by the triangle formed by the center of the Earth and the two pinholes, with the $y$ axis perpendicular to the $z$ axis and pointing away from the center of the Earth. The $x$ axis is defined by the right-hand rule, and is the only horizontal line perpendicular to the $z$ axis.

\section{B. Align the undulator source to the XBA and fiduci- alize the detector}

By putting an x-ray beam through the detector pinhole, we can define the zero $(x=0, y=0)$ of the detector to be the center of the observed x-ray peak. By iteratively adjusting the electron-beam position and slope in the alignment undulator to maximize the $\mathrm{x}$-ray flux through the source pinhole and minimize the beam center deviation from the detector zero, we can bring the undulator x-ray beam centroid to coincide with the XBA [Fig. 1(b)].

\section{Target alignment}

To align a target pinhole to the XBA [Fig. 1(c)], we insert the source pinhole in the undulator $\mathrm{x}$-ray beam to produce a fixed $\mathrm{x}$-ray source, and insert the target pinhole in the beam. We then move the target pinhole with the attached beam line component to center the x-ray beam spot on the detector plane. While the size and transverse stability of the electron beam affects the intensity at the detector plane, the resolution and accuracy of the alignment is determined only by the stability of the optics due to the use of a pinhole as $\mathrm{x}$-ray source.

\section{Alignment accuracy and instrument resolution}

We estimate the alignment accuracy of the target pinholes in three steps:

(i) Calculate the beam spot size and profile at the detector plane produced by a point source.

(ii) Calculate the beam spot size and profile at the detector plane produced by an extended source.

(iii) Convert the measured beam size as the alignment accuracy of the pinhole using the optical geometry.

\section{Pinhole camera resolution at the detector plane}

$\mathrm{X}$-ray pinholes have been used for measuring electronbeam sizes for some years. Their performance is best 


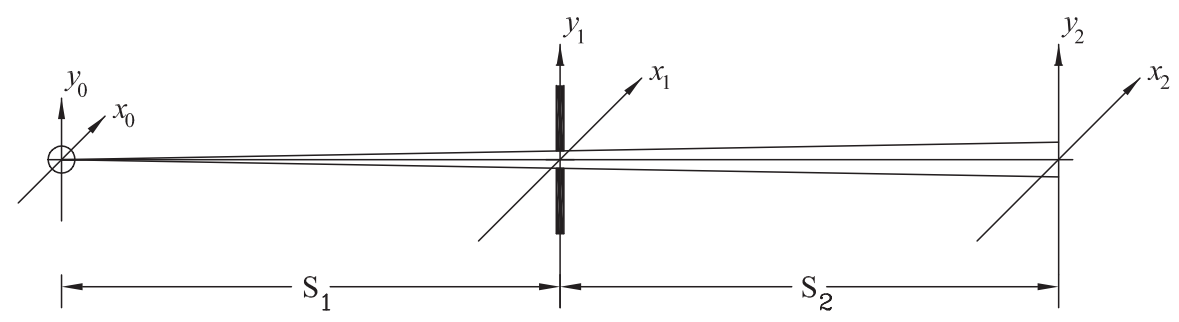

FIG. 2. Schematic of a pinhole camera with a point source. Projection of the pinhole on the image plane shows a magnified area.

modeled by Fresnel diffraction [15-17]. Figure 2 shows a typical geometry of a pinhole camera, with a pinhole located at a distance $S_{1}$ from the source, and an x-ray imaging camera at a distance $S_{2}$ from the pinhole. The Fresnel number, defined as the number of Fresnel zones and given by $F=a_{1}^{2} / \lambda f$, is a key parameter in the model, where $a_{1}$ is the radius of the circular pinhole, $\lambda$ is the wavelength of the light, and the distance $f$ is given by $\frac{1}{f}=\frac{1}{S_{1}}+\frac{1}{S_{2}}$.

In this coordinate system, we label all transverse coordinates on the source plane with subscript 0 , those coordinates on the pinhole plane with subscript 1 , and those coordinates on the image plane with subscript 2 , as shown in Fig. 2. For large Fresnel numbers $(F \gg 1)$ the behavior of the optical system can be approximated by geometric optics. For a point source, the beam spot generated, also known as the point spread function (PSF), on the detector plane is a disc with a radius of $a_{2}=(M+1) a_{1}$, where $M=S_{2} / S_{1}$ is the magnification of the optical system. The annular pattern $I\left(r_{2}\right)$ can be used to derive the integrated $x$ profile:

$$
P\left(x_{2}\right)=\int_{-\infty}^{\infty} I\left(r_{2}\right) d y_{2}=2 \int_{\left|x_{2}\right|}^{\infty} \frac{I\left(r_{2}\right) r_{2}}{\sqrt{r_{2}^{2}-x_{2}^{2}}} d r_{2}
$$

where $r_{2}=\sqrt{x_{2}{ }^{2}+y_{2}^{2}}$ is the radial distance from the axis. The profile is a half-circle and can be fitted to a Gaussian function

$$
P_{\text {fit }}\left(x_{2}\right)=P_{0} e^{-x_{2}^{2} / 2 \sigma_{2, \text { geo }}^{2}}
$$

with a Gaussian width of $\sigma_{2, \text { geo }}=0.59 a_{2}=0.59(M+1) a_{1}$.

For small Fresnel numbers $(F \ll 1)$ the behavior of the optical system can be approximated by the Fraunhofer diffraction, and the PSF is given by the Airy disk, $I(\theta)=I_{0}\left[J_{1}\left(k a_{1} \sin \theta\right) / k a_{1} \sin \theta\right]^{2}$, where the wave vector is given by $k=2 \pi / \lambda$. At a distance $S_{2}$ from the pinhole, the intensity distribution is $I\left(r_{2}\right)=$ $I_{0}\left\{\left[J_{1}\left(k a_{1} r_{2} / S_{2}\right)\right] /\left(k a_{1} r_{2} / S_{2}\right)\right\}^{2}$. Using the same procedure shown in Eqs. (1) and (2), one can derive the Gaussian width for the Fraunhofer diffraction $\sigma_{2 \text {,diff }}=1.31 \lambda S_{2} / a_{1}$. The more accurate Fresnel diffraction PSF can be calcu- lated using the Huygens Principle,

$$
\begin{aligned}
\left|E\left(x_{2}, y_{2}\right)\right|^{2}= & \mid \frac{C}{S_{1} S_{2}} \int \exp \left\{i k \left[\frac{x_{1}^{2}+y_{1}^{2}}{2 S_{1}}\right.\right. \\
& \left.\left.+\frac{\left(x_{1}-x_{2}\right)^{2}+\left(y_{1}-y_{2}\right)^{2}}{2 S_{2}}\right]\right\}\left.d x_{1} d y_{1}\right|^{2} .
\end{aligned}
$$

Its Gaussian profile width can be derived similar to Eqs. (1) and (2). Figure 3 shows the width of the Gaussian profile as a function of $F$. We can see that the Gaussian width approaches the geometric limit for $F \gg 1$ and the Fraunhofer limit for $F \ll 1$. The following expression can be used to approximate the Gaussian width of the Fresnel PSF profiles:

$$
\begin{aligned}
\frac{\sigma_{2, \text { reso }}^{2}}{(M+1)^{2} \lambda f}= & \frac{F}{3}\left(1+\frac{1.5}{F}\right)^{-1} \\
& +0.044\left(0.3+\frac{1}{F}\right), \quad(0.1<F<10) .
\end{aligned}
$$

From Fig. 3, we can also see that for large apertures $(F>0.38)$, the Fresnel diffraction PSF profile is always narrower than that of the geometric projection

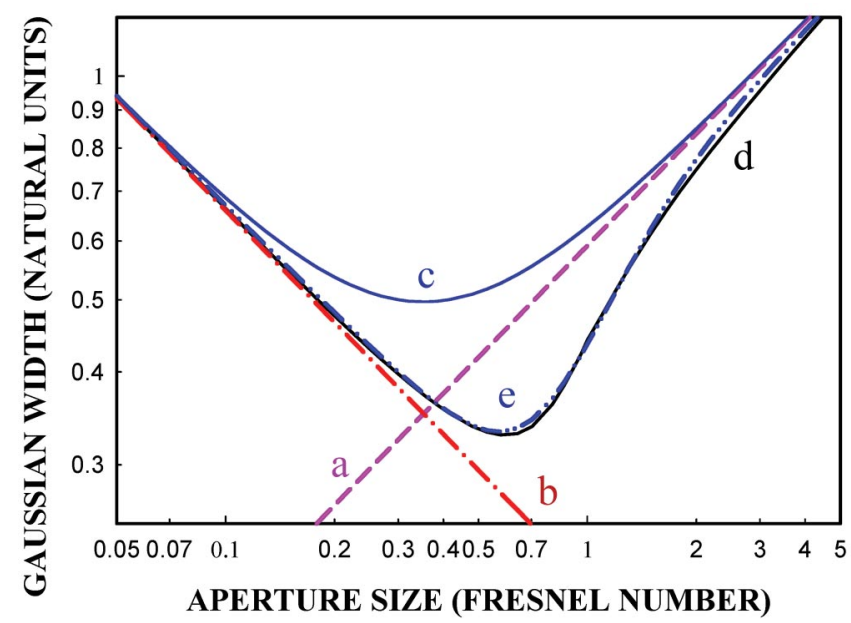

FIG. 3. (Color) Calculated Gaussian width of PSF profiles of a pinhole camera with different models: (a) geometric projection, (b) Fraunhofer diffraction, (c) hybrid model (quadrature sum of the previous two) [18,19], (d) Fresnel diffraction, and (e) fit to (d), as shown in Eq. (4). 


$$
\sigma_{2, \text { reso }} \leq \sigma_{2, \text { geo }}=0.59(M+1) a_{1}, \quad(F \geq 0.38) .
$$

In this regime, we can use the geometric projection for our semiquantitative discussion, with the comforting thought that Fresnel diffraction will only make the beam size smaller.

\section{Beam profile size for an extended source}

When we have an extended source with finite source sizes $\sigma_{x 0}$ and $\sigma_{y 0}$, the beam spot on the detector plane is larger than that of a point source. In this case let us consider the extended source as a collection of point sources in a Gaussian distribution $\rho\left(x_{0}, y_{0}\right)=\rho_{0} e^{-(1 / 2)\left[\left(x_{0}^{2} / \sigma_{x 0}^{2}\right)+\left(y_{0}^{2} / \sigma_{y 0}^{2}\right)\right]}$. For each point source located off axis by $\left(x_{0}, y_{0}\right)$ its beam spot on the detector plane will be centered on $\left(x_{2}, y_{2}\right)=$ $\left(M x_{0}, M y_{0}\right)$, respectively. Summing the contributions of all point sources amounts to calculating the convolution of the Gaussian profile with the pinhole camera PSF profile. The total beam profile sizes at the detector plane are thus

$$
\sigma_{q 2}^{2}=\sigma_{2, \text { reso }}^{2}+M^{2} \sigma_{q 0}^{2}, \quad \text { with }(q=x, y) .
$$

\section{Alignment accuracy of the pinhole position}

We now use the centroid of the beam spot at the detector plane as an indication of whether the pinhole is centered on the optical axis. The error of positioning the centroid of a Gaussian peak is normally a fraction of the peak width, i.e. $\eta \sigma_{q 2}$, where $\eta$ varies between 0.03 and 0.2 , depending on the quality of the photon measurements. Adding the fiducialization error $\eta\left(0.59 a_{D}\right)$ from a detector pinhole of radius $a_{D}$, the total uncertainty is $\eta \sqrt{\sigma_{q 2}^{2}+\left(0.59 a_{D}\right)^{2}}$. For a pinhole displacement of $\Delta x_{1}$, the center of the beam spot on the detector plane moves by

$$
\Delta x_{2}=(M+1) \Delta x_{1} \text {. }
$$

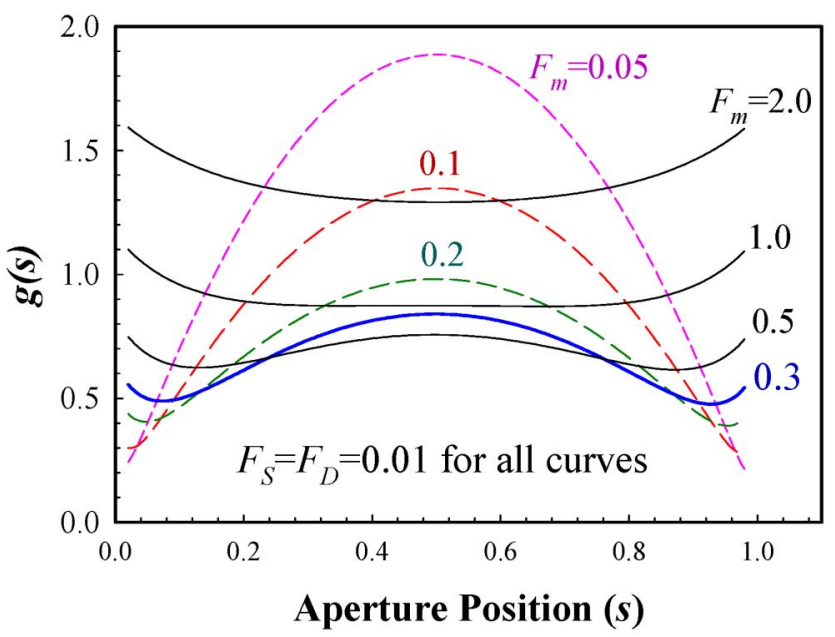

Hence the rms alignment accuracy at the pinhole location is given by

$$
\begin{aligned}
& \delta_{\text {align }}=\frac{\eta}{M+1} \sqrt{\sigma_{q 2}^{2}+0.35 a_{D}^{2}}, \\
& (\eta=0.03-0.2) \text { and }(q=x, y) .
\end{aligned}
$$

Next we introduce a few new variables, the total beam line length $S=S_{1}+S_{2}$, the relative target position $s=$ $S_{1} / S$, the minimum target Fresnel number $F_{m} \equiv 4 a_{1}^{2} / \lambda S$, the scaled source size $F_{S} \equiv 4 a_{S}{ }^{2} / \lambda S$, and the scaled detector size $F_{D} \equiv 4 a_{D}{ }^{2} / \lambda S$. We then rewrite Eq. (8) as

$$
\delta_{\text {align }}=\eta \frac{\sqrt{\lambda S}}{4} g(s), \quad(\eta=0.03-0.2),
$$

where

$$
\begin{aligned}
g(s)= & 1.18\left\{\frac{0.95 F_{m}^{2}}{F_{m}+6 s(1-s)}+0.15 s(1-s)\right. \\
& \left.+\frac{2}{F_{m}} s^{2}(1-s)^{2}+(1-s)^{2} F_{S}+s^{2} F_{D}\right\}^{1 / 2} .
\end{aligned}
$$

The left graph of Fig. 4 shows the function $g(s)$ for a near-zero source with size $F_{S}=F_{D}=0.01$. It can be seen that when $F_{m}$ is in the range of $0.3-0.45$, the Fresnel diffraction pattern is at its minimum width for most of the beam line, and a good resolution is obtained over the entire beam line. The Fig. 4 right graph shows the dependence of the function $g(s)$ on the source size for a fixed, near-ideal target size $\left(F_{m}=0.35\right)$. It can be seen that when $F_{S}$ and $F_{D}$ are in the range of $0.3-0.5$, the function $g(s)$ is fairly flat and less than one. In short, the ideal operating condition can be represented as

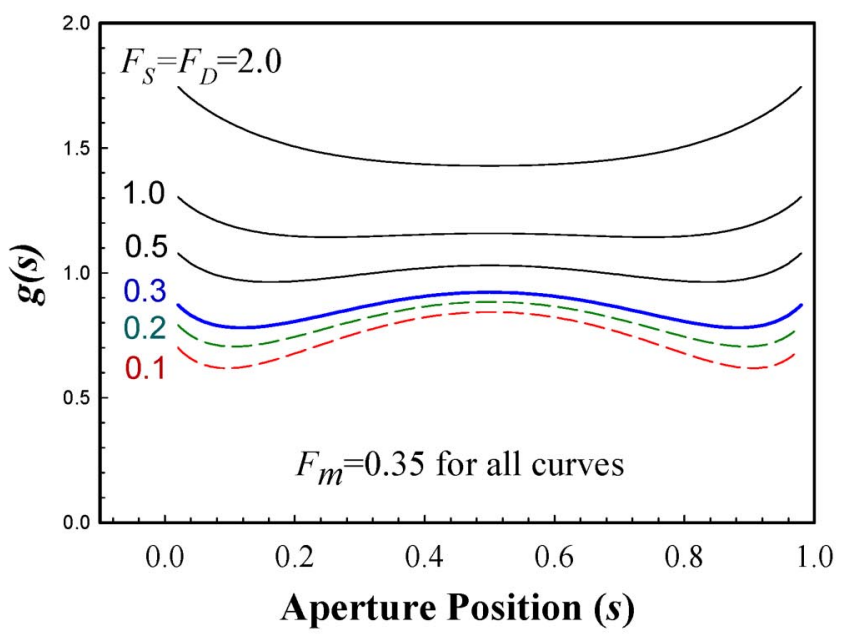

FIG. 4. (Color) Function $g(s)$ for different aperture sizes with near-zero source size ( $F_{S}=F_{D}=0.01$, left graph). Function $g(s)$ for different source sizes with near-ideal target size $\left(F_{m}=0.35\right.$, right graph). 


$$
\left.\left.\begin{array}{l}
F_{S}=0.50 \pm 0.10 \\
F_{m}=0.36 \pm 0.05 \\
F_{D}=0.50 \pm 0.10
\end{array}\right\}, \quad \text { or } \quad \begin{array}{l}
a_{S}=(0.35 \pm 0.04) \sqrt{\lambda S} \\
a_{1}=(0.30 \pm 0.02) \sqrt{\lambda S} \\
a_{D}=(0.35 \pm 0.04) \sqrt{\lambda S}
\end{array}\right\}
$$

with the resulting alignment accuracy of

$$
\delta_{\text {align }} \approx \eta(\sqrt{\lambda S} / 4), \quad(\eta=0.03-0.2) .
$$

In the case of the LCLS undulator, for example, we use the following parameters [20]: $\lambda=0.5 \AA, S=200 \mathrm{~m}$, and $\eta=0.1$. The ideal target pinhole radius is $30 \pm 2(\mu \mathrm{m})$, while the radii for the source and detector pinholes are $35 \pm 4(\mu \mathrm{m})$. The estimated positioning accuracy according to Eq. (12) is

$$
\delta_{\text {align }} \leq 0.1 \times 25(\mu \mathrm{m})=2.5(\mu \mathrm{m}) .
$$

\section{X-RAY OPTICS ISSUES}

In this section, we will discuss how to choose specific pinhole materials, tailor the detector system response, and specify the undulator source for precision alignment.

\section{A. Pinhole}

The intensity of a monochromatic $\mathrm{x}$-ray beam attenuates exponentially into a uniform medium [21]. The fraction of flux transmitted through a foil of thickness $t$ is given by $\alpha_{t}=e^{-\mu_{M} \rho_{M} t}$, where $\mu_{M}$ is the mass attenuation coefficient and $\rho_{M}$ is the density of the metal. We note that a pinhole made with a semitransparent foil will have a peakto-background ratio of $\sim 1 / \alpha_{t}$ immediately downstream of the pinhole.

In the last section, we noted that if we are using the optimum aperture size, the x-ray beam spot on the detector plane is usually narrower than that of the geometric projection of the pinhole aperture, and the profile peak is higher than that of the geometric projection. Hence for estimating the signal-to-background ratios, we can safely use the approximation of the geometric projection, where the ratio is preserved downstream of the aperture. A simple rule of thumb can be given as $\alpha_{t} \leq\left(1 / r_{S / B}^{(\min )}\right)$, where $r_{S / B}^{(\min )}$ is the minimum signal-to-background ratio the experiment needs. The above two expressions can be combined to give

$$
t \geq t_{\min }\left(r_{S / B}^{(\min )}\right) \equiv\left(\mu_{M} \rho_{M}\right)^{-1} \ln r_{S / B}^{(\min )}
$$

where $t_{\min }$ is the minimum thickness for the pinhole foil. Table I shows the minimum foil thickness for several desirable signal-to-background ratios. From the same table we can see that if we accept a background level of $10 \%$, then a 0.5 -mm-thick foil ( $\mathrm{Ta}, \mathrm{W}$, or $\mathrm{Au}$ ) would be sufficient for applications using photon energy up to $110 \mathrm{keV}$. Similarly, a 0.25 -mm-thick foil would be sufficient for applications using photon energy up to $50 \mathrm{keV}$. These requirements can be further relaxed for polychromatic sources. For example, for an x-ray source with a nearly uniform spectrum in the region of 10 to $100 \mathrm{keV}$, a 0.25 mm-thick foil would likely be sufficient since most lowenergy photons will be blocked by the foil. Finally, we see from the example in the previous section that suitable pinholes for target and source apertures have diameters of $50 \mu \mathrm{m}$ or larger. Hence the pinhole aspect ratio is less than ten. The fabrication of these pinholes is well within today's technological capabilities.

\section{B. Detector system}

For x-ray alignment we can use imaging and nonimaging detectors. Imaging detectors use two-dimensional arrays to acquire a map of the photon flux. Since most types of pixilated x-ray detectors are susceptible to radiation damage at high flux, the popular approach is to use an $\mathrm{x}$ ray scintillator (YAG, for example) to convert the $\mathrm{x}$ rays to visible light and then utilize optical lenses and cameras to acquire the image. The nonimaging detectors are commonly referred to as position-sensitive detectors (PSDs). While many designs exist, the majority of them operate on the principle of dividing the signal of the detector into several parts or quadrants, with their fractions of the signal dependent on the position of the beam centroid, such that the beam position is proportional to the ratio of the difference between the two opposing parts over their sum signal.

\begin{tabular}{|c|c|c|c|}
\hline $\begin{array}{l}\text { Minimum } \\
\text { Signal/ } \\
\text { Background } \\
\text { ratio }\end{array}$ & $\begin{array}{c}\text { Tantalum }(\mathrm{Ta}) \\
\left(\text { density }=16.8 \mathrm{~g} / \mathrm{cm}^{3}\right) \\
\left(\mu_{M} \rho_{M}\right)^{-1}=0.218 \mathrm{~mm} \\
\text { at the K-edge } 66.70 \mathrm{keV}\end{array}$ & $\begin{array}{c}\text { Tungsten }(\mathrm{W}) \\
\left(\text { density }=19.25 \mathrm{~g} / \mathrm{cm}^{3}\right) \\
\left(\mu_{M} \rho_{M}\right)^{-1}=0.202 \mathrm{~mm} \\
\text { at the K-edge } 69.33 \mathrm{keV}\end{array}$ & $\begin{array}{c}\text { Gold }(\mathrm{Au}) \\
\left(\text { density }=19.32 \mathrm{~g} / \mathrm{cm}^{3}\right) \\
\left(\mu_{M} \rho_{M}\right)^{-1}=0.232 \mathrm{~mm} \\
\text { at the K-edge } 69.33 \mathrm{keV}\end{array}$ \\
\hline 3 & $0.25 \mathrm{~mm}$ & $0.22 \mathrm{~mm}$ & $0.25 \mathrm{~mm}$ \\
\hline 5 & $0.35 \mathrm{~mm}$ & $0.33 \mathrm{~mm}$ & $0.38 \mathrm{~mm}$ \\
\hline 10 & $0.50 \mathrm{~mm}$ & $0.47 \mathrm{~mm}$ & $0.53 \mathrm{~mm}$ \\
\hline 100 & $1.00 \mathrm{~mm}$ & $0.93 \mathrm{~mm}$ & $1.06 \mathrm{~mm}$ \\
\hline 1000 & $1.51 \mathrm{~mm}$ & $1.36 \mathrm{~mm}$ & $1.59 \mathrm{~mm}$ \\
\hline
\end{tabular}
The resolution of the PSD is often a small fraction of the beam size, usually on the order of 1 to several per cent. Unlike imaging detectors, a PSD cannot pick out a narrow peak from a wide background, and hence is more susceptible to background noise.

TABLE I. Required thickness of selected metal foils for minimum signal-to-background ratio (x-ray photon energy $<100 \mathrm{keV}$ ). 
TABLE II. Calculated mirror constant $\mathrm{C}$ for selected metals (bulk density assumed).

\begin{tabular}{cccc}
\hline \hline Metal & $\mathrm{Re}$ & $\mathrm{Au}$ & $\mathrm{Pt}$ \\
\hline Mirror constant (keV-mrad) & 66 & 80 & 85 \\
\hline \hline
\end{tabular}

A detector responding to only a narrow band of x-ray wavelength is often useful in improving the signal-tobackground ratio. Filters made of low- $Z$ material $(\mathrm{Be}, \mathrm{C}$, $\mathrm{Al}$, and $\mathrm{Si}$ ) can be placed upstream of the detector to remove unwanted long wavelength photons and stray light. On the other hand, mirrors coated with high- $Z$ metal can also be used to remove unwanted high-energy photons. As a rule of thumb, the cut-off photon energy can be written as $\omega_{c} \approx C / \theta$, where $\theta$ is the grazing incidence angle in mrad, and $C$ is a material-dependent constant, several of which are listed in Table II. An alternative approach, though less effective, is to reduce the detector's response to highenergy photons by making the detector from thin low- $Z$ material, near or below two absorption lengths at the working photon energy.

\section{X-ray sources}

X-ray alignment over long distances requires a highly brilliant source. Take, for example, the LCLS, where we chose a $70-\mu \mathrm{m}$ source pinhole and a $60-\mu \mathrm{m}$ target pinhole located at $100 \mathrm{~m}$ from the source. In order to obtain an image with $\sim 10^{6}$ photons in $1 \mathrm{~s}$, a source brilliance of $\sim 10^{15}$ photons $/ \mathrm{mm}^{2} / \mathrm{mrad}^{2}$ is required. This brilliance is comparable to a modern bending magnet source with a $\sim 0.2 \mathrm{~mA}$ electron current, or an undulator source with a $20 \mathrm{nA}$ average current.

For a linac-based facility with an average current of 10$100 \mathrm{nA}$, the only practical x-ray source for alignment is an undulator. An undulator has a fundamental frequency $\omega_{1} \equiv 4 \pi c \gamma^{2} / \lambda_{u}\left(1+K^{2} / 2\right)$, where $\lambda_{u}$ is the magnetic period length of the undulator, $\gamma$ is the Lorentz factor of the electron, and $K$ is the undulator magnetic parameter. On the beam axis, the photons aggregate in regions near odd multiples of $\omega_{1}$ [21-23], and the on-axis photon spectrum is a narrow spike that can be expressed as a sinc function. For x-ray alignment applications, it is sufficient to consider the undulator source spectrum as a sum of delta functions:

$$
\begin{aligned}
F(\omega)= & 174.4 s_{0} N E^{2}[\mathrm{GeV}] Q[n C] \Delta \Omega\left[(\mu \mathrm{rad})^{2}\right] \\
& \times \sum_{m=1}^{\infty} \frac{f_{2 m-1}(K)}{2 m-1} \delta\left[\omega-(2 m-1) \omega_{1}\right]
\end{aligned}
$$

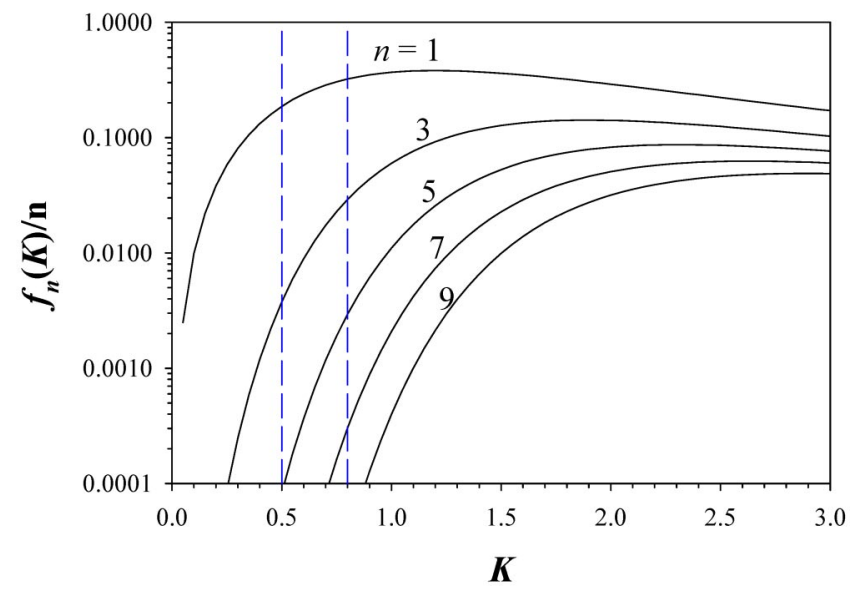

FIG. 5. (Color) Function $f n(K) / n$ for the first five odd harmonics.

where

$$
\begin{aligned}
f_{n}(K)= & \frac{n^{2} K^{2}}{\left(1+K^{2} / 2\right)^{2}} \\
& \times\left\{J_{(n-1) / 2}\left[\frac{n K^{2}}{4\left(1+K^{2} / 2\right)}\right]\right. \\
& \left.-J_{(n+1) / 2}\left[\frac{n K^{2}}{4\left(1+K^{2} / 2\right)}\right]\right\}^{2}, \quad(n=1,3, \ldots) .
\end{aligned}
$$

The source pinhole transmission factor $s_{0}=$ $1-e^{-a_{S}^{2} / 2 \sigma_{0}^{2}}$ represents the shadowing effect of the source pinhole where only a fraction of x-ray photons are able to pass through to reach the target pinhole. In this case $\sigma_{0}$ represents the rms radius of the electron beam.

Figure 5 shows the function $f_{n}(K) / n$ for the first five odd harmonics. We note that if we choose $0.5 \leq K \leq 0.8$, the first harmonic flux is over $50 \%$ of its peak, the third harmonic is at (2-9)\% of the intensity of the first harmonic, and the fifth harmonic is at the (0.04-0.9)\% level. Furthermore, if we let the third harmonic photon energy fall above the absorption edge of the pinhole material, only the fifth and higher harmonics may penetrate the pinhole foil to produce background. Hence we will be able to use thinner foils $(0.25 \mathrm{~mm})$ to make pinholes. Table III summarizes our recommendation for an LCLS alignment undulator with a clean spectrum.

TABLE III. Parameters of a clean undulator for x-ray alignment.

\begin{tabular}{lccc}
\hline \hline \multicolumn{1}{c}{ Pinhole material } & Ta & W & Au \\
\hline First harmonic energy $\hbar \omega_{1}$ & $\geq 22.6 \mathrm{keV}$ & $\geq 23.3 \mathrm{keV}$ & $\geq 26.6 \mathrm{keV}$ \\
Magnetic parameter $K$ & $0.5-0.8$ & $0.5-0.8$ & $0.5-0.8$ \\
\hline \hline
\end{tabular}




\section{Example: A dedicated LCLS alignment undulator}

In this example, we consider the LCLS undulator with a dedicated alignment undulator upstream of the tune-up dump, which can be removed from the beam by way of opening its gap. Table IV shows the hardware parameters and expected performance data. Note that the electron energy and magnetic gap value are calculated to meet the requirements for $K$ and $\omega_{1}$.

\section{FIDUCIALIZATION ISSUES}

So far we have established a procedure to align x-ray pinholes to a long straight line at very high accuracy. The technique would not be very useful unless we can relate the pinholes to beam line components and their functioning center points, center lines, or center planes with equal accuracy. In this section, we will address fiducialization techniques using conventional laboratory instruments or offline fiducialization and methods using the electron or $\mathrm{x}$ ray beam for in situ or online fiducialization.

\section{A. Offline fiducialization}

It is common practice that prior to the installation of accelerator components the relationship between the beam axis and outside reference markers be established in a fiducialization step. During the girder assembly process these markers are then used to create the proper relative position between individual elements without having direct access to a representation of the beam axes. In some cases, such as the insertion device vacuum chamber, an accessible reference surface is used for positioning, after quality control measurements have confirmed its accuracy and relation to the mechanical beam axes. Other components such as quadrupoles and undulators require an intermediate step to establish the magnetic axes that will be used as reference for determining the location of the fiducial markers. This step adds another level of uncertainty due to probe noise at low field values in determining the position accuracy of these components.

Well-known techniques are used to establish the magnetic axes of quadrupoles and undulators, while state-ofthe-art coordinate measurement systems provide the accuracy and positioning control in the assembly step. In order to realize tight position tolerances, many mechanical effects, especially repeatability issues of actuators, have to be taken into consideration. The online fiducialization techniques outlined below will provide a direct link between the XBA and the EBA, and circumvent some of these mechanical issues.

TABLE IV. Design parameters for LCLS x-ray alignment with a dedicated undulator source.

\section{Electron beam}

\begin{tabular}{|c|c|}
\hline Electron energy & $10.66 \mathrm{GeV}$ \\
\hline Bunch charge & $1.0 \mathrm{nC}$ \\
\hline Electron-beam size & $45 \mu \mathrm{m}$ \\
\hline \multicolumn{2}{|l|}{ Alignment undulator } \\
\hline Period & $3.3 \mathrm{~cm}$ \\
\hline Number of periods & 72 \\
\hline Magnetic gap & $23 \mathrm{~mm}$ \\
\hline Magnetic parameter $K$ & 0.8 \\
\hline First harmonic photon energy $\hbar \omega_{1}$ & $24.8 \mathrm{keV}$ \\
\hline \multicolumn{2}{|l|}{$\mathrm{X}$-ray pinholes } \\
\hline Pinhole foil material & Tantalum or tungsten \\
\hline Pinhole foil thickness & $0.25 \mathrm{~mm}$ \\
\hline Source pinhole diameter $2 a_{0}$ & $60 \mu \mathrm{m}$ \\
\hline Target pinhole diameter $2 a$ & $70 \mu \mathrm{m}$ \\
\hline Detector pinhole diameter & $70 \mu \mathrm{m}$ \\
\hline Source pinhole transmission factor $s_{0}$ & $83 \%$ \\
\hline \multicolumn{2}{|l|}{ Photon flux through target pinhole @ 100 m } \\
\hline First harmonic flux, $F_{1}{ }^{(\mathrm{O})}$ & $7.7 \times 10^{4}(\mathrm{ph} / \mathrm{shot})$ \\
\hline Third harmonic flux, $F_{3}(\mathrm{O})$ & $0.7 \times 10^{4}(\mathrm{ph} / \mathrm{shot})$ \\
\hline Fifth harmonic flux, $F_{5}(\mathrm{O})$ & $700(\mathrm{ph} / \mathrm{shot})$ \\
\hline \multicolumn{2}{|l|}{ Detector } \\
\hline Region of interest & $8 \mathrm{~mm} \times 8 \mathrm{~mm}$ \\
\hline Filter thickness/material & $0.1 \mathrm{~mm} /$ silicon \\
\hline Mirror & None \\
\hline Scintillator material & YAG \\
\hline Scintillator thickness & $1 \mathrm{~mm}$ \\
\hline Detector efficiency at $\hbar \omega_{1}$ & $74 \%$ \\
\hline Target alignment accuracy (rms) & $2.5 \mu \mathrm{m}$ or better \\
\hline
\end{tabular}




\section{B. Online fiducialization}

As the bench fiducialization cannot provide sufficient accuracy for the most stringent applications, especially for magnetic components where the conditions on the bench, such as temperature, magnet orientation, stray magnetic fields, etc., may vary from those found on the beam line, we need to develop online positioning schemes to provide the required accuracy. Beam line components interacting with x-ray photons, such as wire scanners and fluorescent screens, can be directly positioned with the alignment $\mathrm{x}$ ray beam (direct positioning). Other components interacting only with electrons, such as beam position monitors (BPMs) and optical transition radiation (OTR) screens, will have to be positioned with the electron beam as the transfer medium, after the position of the latter has been measured (indirect positioning). Since fluorescent screens interact with both electron and x-ray beams and are compatible with single-shot operation, they provide a convenient link between the two positioning classes. As a consequence, the transfer error of the screen will be inherited by all indirectly positioned components. Well-built, highly reproducible wire scanners can also be used to measure the electron-beam position relative to the XBA. While not compatible with single-shot measurement, their ability to detect the electron beam in a background of $\mathrm{x}$ rays using downstream bremsstrahlung radiation makes them uniquely suitable inside undulators and free-electron lasers. In the following, the online positioning process of a fluorescent screen, a wire position monitor, and electronbeam position monitors are described in more detail. The positioning accuracy of other components, such as quadrupoles and OTR screens can be similarly derived [24].

\section{Fiducialization of downstream fluorescent screens in proximity of target pinholes}

For fluorescent screens with a highly reproducible drive mechanism or a reliable encoder, it is only necessary to find the location "zero" on the image plane where the XBA intercepts the screen. The following procedure, also shown in Fig. 6 outlines the steps to locate this point:

(i) Find an upstream target pinhole near the fluorescent screen and position it on the XBA using the target alignment procedure discussed in Sec. II C.

(ii) Insert the fluorescent screen and record the image of the x-ray beam on the camera. The center of the beam spot can then be taken as the zero position for the screen. The fiducialization accuracy can be estimated to be

$$
\delta_{F S} \approx \sqrt{\delta_{\text {align }}^{2}+\delta_{F S, \text { trans }}^{2}},
$$

where $\delta_{\text {align }}$ is the alignment accuracy of the target pinhole itself, and $\delta_{F S \text {,trans }}$ is the rms transfer error for fiducializing the fluorescent screen and is given by

$$
\delta_{F S, \text { trans }} \approx \sqrt{\eta_{x}^{2}\left(0.35 a_{1}^{2}+\sigma_{\text {res }, x}^{2}\right)+\delta_{F S, \text { rep }}^{2}},
$$

where $\sigma_{\text {res }, x}$ is the resolution of the camera/optics for x-ray beam excitation, and $\delta_{F S \text {,rep }}$ is the rms error related to the mechanical reproducibility of the screen insertion mechanism. The constant $\eta_{x}$ depends on the quality of the measurement and generally is better for higher flux. If we are using the screen immediately after fiducialization for centering the e-beam at the same location without moving the insertion mechanism, the last term in Eq. (18) can be omitted.

\section{Fiducialization of wire scanners in proximity of target pinholes}

For wire scanners with a highly reproducible drive mechanism or a reliable encoder, it is only necessary to find the location zero where the wire element crosses the XBA. Figure 7 shows a procedure to locate this point.

(i) First find a target pinhole near the scanner and position it on the XBA using the target alignment procedure discussed in Sec. II C.

(ii) Next the wire can be scanned across the x-ray beam. The wire partially shadows the beam when passing through the small $\mathrm{x}$-ray beam, producing a reduction in $\mathrm{x}$-ray intensity seen by the x-ray detector at the end of the beam line. The point of maximum reduction can thus be taken as the zero position for the wire scanner. The fiducialization accuracy can be estimated to be

$$
\delta_{W S} \approx \sqrt{\delta_{\text {align }}^{2}+\delta_{W S \text {,trans }}^{2}},
$$

where $\delta_{\text {align }}$ is the alignment accuracy of the target

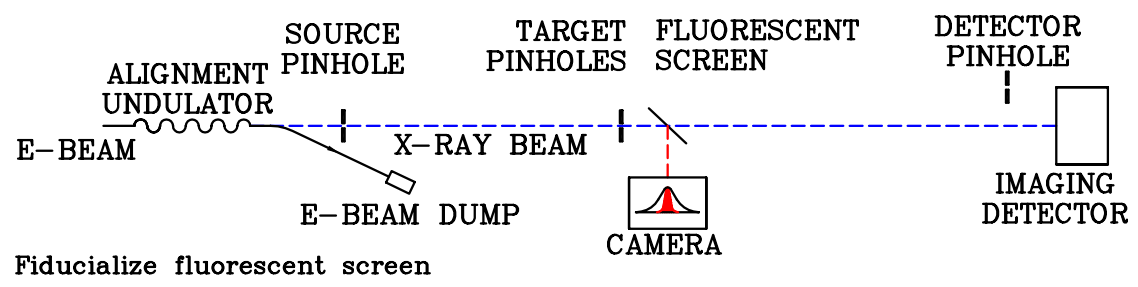

FIG. 6. (Color) Fiducialization of a fluorescent screen near a target pinhole by taking the x-ray beam image on the fluorescent screen after aligning the $\mathrm{x}$-ray pinhole. 


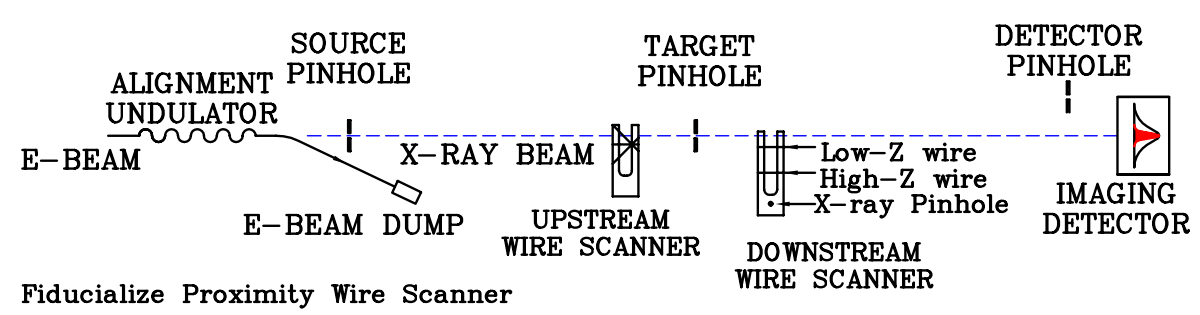

FIG. 7. (Color) The upstream wire scanner demonstrates its fiducialization near a target pinhole using $\mathrm{x}$ rays by scanning the wire across the $\mathrm{x}$-ray beam after aligning the $\mathrm{x}$-ray pinhole. The downstream wire scanner demonstrates that either an integral high- $Z \mathrm{x}$-ray wire or an $\mathrm{x}$-ray pinhole with known distance from the low- $Z$ electron-beam wire can be used as $\mathrm{x}$-ray beam fiducialization markers.

pinhole itself, and $\delta_{W S \text {,trans }}$ is the rms transfer error for fiducializing the wire scanner and is given by

$$
\delta_{W S \text {, trans }} \approx \sqrt{0.35 \eta_{W S}^{2}\left(a_{1}^{2}+R^{2}\right)+\delta_{W S, \text { rep }}^{2}},
$$

where $a_{1}$ and $R$ are the radii of the target pinhole and wire, respectively, and $\delta_{W S \text {,rep }}$ is the rms error related to the mechanical reproducibility of the scanning mechanism. The constant $\eta_{W S}$ depends on the quality of the measurement and generally is better for high- $Z$ wires. For wires made of low- $Z$ material, such as carbon, the above fiducialization technique is not very effective since the wire is nearly transparent to the $\mathrm{x}$-ray beam. In that case an alternative is to attach a high- $Z$ x-ray wire or an $\mathrm{x}$-ray pinhole directly to the wire scanner as a fiducialization marker, and precisely measure the distance between the wire and marker prior to installation of the scanner. Hence when the fiduciali- zation marker is aligned to the XBA, the wire is at a known position. Unlike the fluorescent screens, this fiducialization procedure is usable for wire scanners both upstream and downstream of the target pinhole.

\section{Fiducialization of electron-beam position monitors (BPMs, indirect)}

A high-resolution electron BPM usually has good linearity within its dynamic range, but its electric center may not coincide with its mechanical center, or with the XBA. The following fiducialization procedure directly determines the offset difference between the electric center and the XBA, thus bypassing the need to know the mechanical center of the BPM. We will fiducialize the BPMs in two stages: In the first stage, we only fiducialize BPMs with a pinhole-fluorescent screen combination immediately downstream of them, using the fluorescent screen as

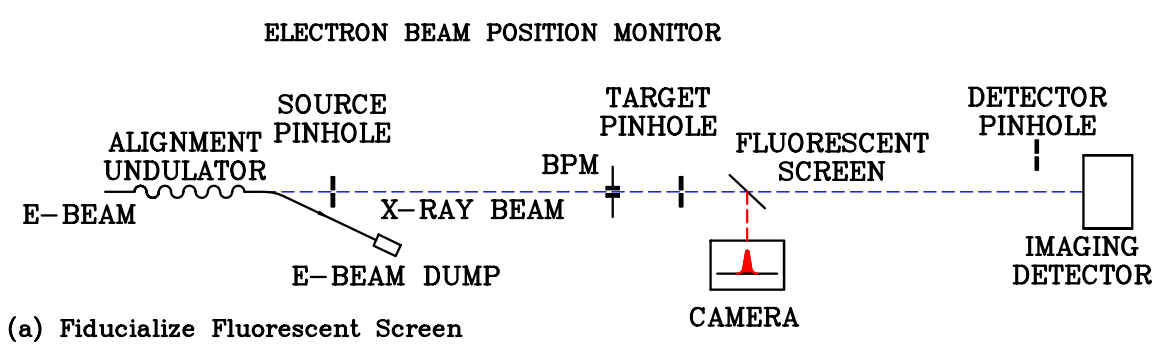

(a) Fiducialize Fluorescent Screen
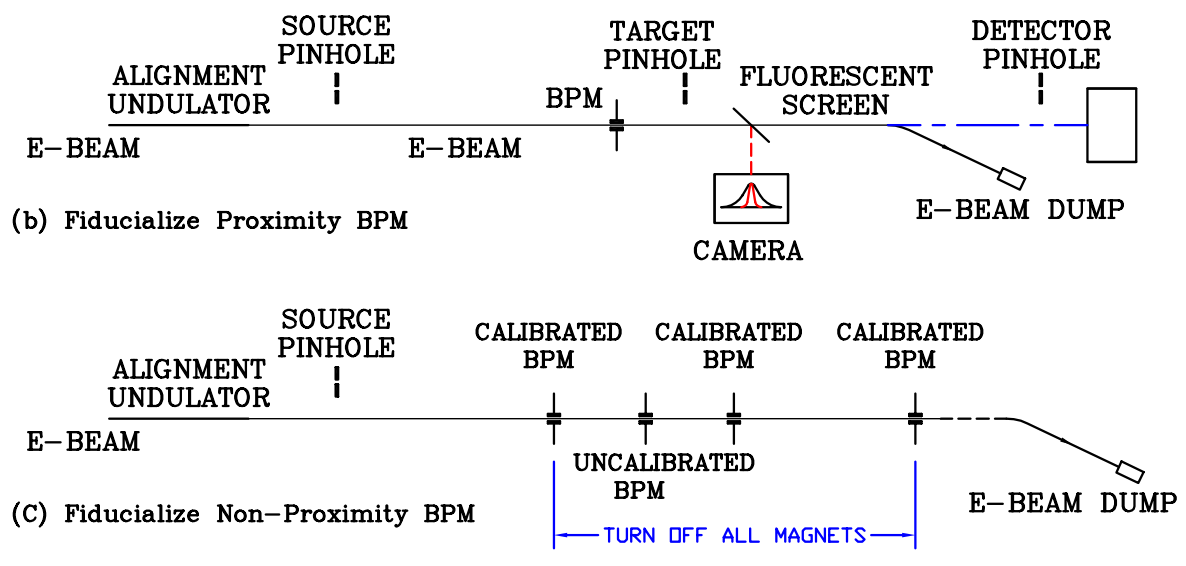

FIG. 8. (Color) Fiducialization of electron BPMs. 
the electron-beam position monitor. In the second stage, we will create field-free regions between fiducialized BPMs and use them to determine the electron-beam position and offsets of other BPMs in the region.

Fiducializing BPMs near a fluorescent screen.-We measure the offsets of BPMs located immediately upstream of a pinhole-fluorescent screen combination in three steps:

(i) Align the x-ray pinhole (Sec. IIC).

(ii) Fiducialize the fluorescent screen downstream of the BPM using the x-ray beam (Sec. IV B 1 and Fig. 8(a)). (iii) Remove all pinholes, open the undulator gap, let the electron beam down the beam line, and position the electron beam's charge center on the fiducialized center of the fluorescent screen [Fig. 8(b)].

The reading on the BPM is the offset. The fiducialization accuracy can be estimated to be

$$
\delta_{\mathrm{BPM}} \approx \sqrt{\delta_{\text {align }}^{2}+\delta_{B P M, \text { trans }}^{2}}
$$

where the rms transfer error for fiducializing the BPM $\delta_{\mathrm{BPM}, \text { trans }}$ is given by

$$
\delta_{\mathrm{BPM}, \text { trans }} \approx \sqrt{\eta_{x}^{2}\left(0.35 a_{1}^{2}+\sigma_{\mathrm{res}, x}{ }^{2}\right)+\eta_{e}{ }^{2}\left(\sigma_{e}{ }^{2}+\sigma_{\mathrm{res}, e}{ }^{2}\right)+\sigma_{\mathrm{BPM}}{ }^{2}}
$$

where $\sigma_{\mathrm{BPM}}$ is the rms resolution of the electron BPM, $\sigma_{e}$ is the electron-beam radius, $\sigma_{\text {res }, e}$ is the resolution of the camera/optics for electron-beam excitation, and $\eta_{e} \sqrt{\sigma_{e}^{2}+\sigma_{\text {res, } e}^{2}}$ is the accuracy for determining the electron-beam center from the screen image. Similarly the term $\eta_{x} \sqrt{0.35 a_{1}^{2}+\sigma_{\text {res }, x}^{2}}$ represents the accuracy for determination of the x-ray beam center from the screen image. In this case we have omitted the mechanical insertion error since we do not move the screen between $\mathrm{x}$-ray fiducialization and the electron-beam measurement.

Fiducializing BPMs far away from fluorescent screens. - Strictly speaking, the $\mathrm{x}$-ray alignment technique cannot be used to fiducialize BPMs far away from fluorescence screens or wire scanners. We propose to use a variation of the ballistic alignment technique [25] to fiducialized electron BPMs. In this case we first turn off all magnets in a section of the accelerator containing several already fiducialized BPMs.

(i) In the lowest order approximation, when the section is shielded by soft steel from ambient fields, including the Earth's magnetic field, we may take the electron trajectory as a straight line. Only two already calibrated BPMs are needed to determine the electron trajectory. The offsets of other BPMs in the field-free regions can be determined using ballistic alignment.

(ii) In the first order approximation, the Earth's field and ambient fields are taken to be uniform; three fiducialized BPMs are needed to calculate the curved trajectory in the region. The offset of other BPMs in the region can be determined using the calculated trajectory.

(iii) In the next higher order approximation, the Earth's field and ambient field are assumed to have a linear slope; four fiducialized BPMs can be used to calculate the curved trajectory in the region. The approximation can be improved as more and more fiducialized BPMs are used.

In reality, however, the accelerator and the tunnel contain many lumped magnetic components that make the above approaches poor approximations. In this case the fiducialization of these BPMs is likely to be less accurate, depending on the site conditions. As we pointed out earlier, an improvement in this area will only come from better electron-beam technology and additional magnetic shielding.

\section{Alignment of magnetic components}

In most accelerator designs, a BPM is normally positioned near a quadrupole magnet. Since we have determined the electron-beam position when it coincides with the XBA at the BPM, we can hold that position of the electron beam and move the nearby quadrupole magnet to search for its center. The magnet center deviation from the XBA can be detected with one of the approaches used for the electron-beam-based alignment (EBBA) process [11,12]:

(i) Scan the strength of the magnet and watch for downstream trajectory changes.

(ii) Vary the energy of the electron beam and watch for downstream trajectory changes.

(iii) Mechanically scan the magnet and watch for a zero-deflection in downstream trajectory changes.

Other FEL components such as planar undulators are periodic magnetic dipole structures that require precision alignment especially in the vertical direction. In the plane bisecting the undulator gap, the periodic magnetic field has minimum amplitude. The amplitude then increases quadratically with the distance from the center plane. This increase in magnetic field induces a spectrum shift in the spontaneous radiation. By moving the electron trajectory relative to the undulator, a spatial positioning resolution on the order of $10 \mu \mathrm{m}$ can be obtained using appropriate $\mathrm{x}$-ray 
TABLE V. RMS XBBA accuracy for LCLS undulator components.

\begin{tabular}{|c|c|c|c|c|}
\hline Component & Formula for the accuracy estimate & Transfer error & Alignment accuracy & Notes \\
\hline Target pinhole & $\delta_{\text {align }} \approx \eta(\sqrt{\lambda S} / 4)$ & None & $2.5 \mu \mathrm{m}$ & $\begin{array}{c}a_{1}=30 \mu \mathrm{m}, a_{0}=30 \mu \mathrm{m}, \\
\quad a_{D}=30 \mu \mathrm{m}, \\
\eta=0.1, \lambda=0.5 \AA \\
\text { and } S=200 \mathrm{~m}\end{array}$ \\
\hline Wire scanner & $\begin{array}{c}\delta_{W S} \approx \sqrt{\delta_{\text {align }}^{2}+\delta_{W S \text { trans }}^{2}} \\
\delta_{W S, \text { trans }} \approx \sqrt{0.35 \eta_{W S}^{2}\left(a_{1}^{2}+R^{2}\right)+\delta_{W, \text { rep }}^{2}}\end{array}$ & $2.0 \mu \mathrm{m}$ & $3.2 \mu \mathrm{m}+\mathrm{mech}^{\mathrm{a}}$ & $\begin{array}{c}R=15 \mu \mathrm{m} \\
\text { other values as } \\
\text { above }\end{array}$ \\
\hline Fluorescent screen & $\begin{aligned} & \delta_{F S} \approx \sqrt{\delta_{\text {align }}^{2}+\delta_{F S, \text { trans }}^{2}} \\
\delta_{F S \text {,trans }} \approx & \sqrt{\eta_{x}^{2}\left(0.35 a_{1}^{2}+\sigma_{\text {res }, x}^{2}\right)+\delta_{F S, \text { rep }}^{2}}\end{aligned}$ & $2.0 \mu \mathrm{m}$ & $3.2 \mu \mathrm{m}+\mathrm{mech}^{\mathrm{a}}$ & $\begin{array}{c}\sigma_{\text {res }, x}=10 \mu \mathrm{m}, \\
\text { other values as } \\
\text { above }\end{array}$ \\
\hline BPM & $\delta_{\mathrm{BPM}, \text { trans }} \approx \sqrt{\eta_{x}{ }^{2}\left(0.35 a_{1}^{2}+\sigma_{\mathrm{BPS}, x}{ }^{2}\right)+\eta_{e}{ }^{2}\left(\sigma_{e}{ }^{2}+\sigma_{\mathrm{res}, e}{ }^{2}\right)+\sigma_{\mathrm{BPM}}^{2}}$ & $3.8 \mu \mathrm{m}$ & $4.5 \mu \mathrm{m}$ & $\begin{array}{c}\sigma_{\mathrm{res}, e}=40 \mu \mathrm{m} \\
\sigma_{e}=45 \mu \mathrm{m} \\
\sigma_{\mathrm{BPM}}=1 \mu \mathrm{m} \\
\eta_{e}=0.05 \\
\text { other values as } \\
\quad \text { above }\end{array}$ \\
\hline OTR screen & $\delta_{\text {OTR }, \text { trans }} \approx \sqrt{\delta_{\text {align }}^{2}+\delta_{\mathrm{OTR}, \text { trans }}^{2}}$ & $6.0 \mu \mathrm{m}$ & $6.5 \mu \mathrm{m}+\mathrm{mech}^{\mathrm{a}}$ & $\begin{array}{c}\sigma_{\text {res, OTR }}=10 \mu \mathrm{m} \\
\sigma_{e}=45 \mu \mathrm{m} \\
\text { other values as } \\
\text { above }\end{array}$ \\
\hline Quadrupole magnet & $\begin{array}{c}\delta_{\text {quad }} \approx \sqrt{\delta_{\text {align }}^{2}+\delta_{\text {quad,trans }}^{2}} \\
\delta_{\text {quad,trans }} \approx \sqrt{\delta_{\mathrm{BPM}, \text { trans }}^{2}+\left(\frac{3.3 E}{\varepsilon_{Q} \beta Q_{0}} \sigma_{\mathrm{BPM}}\right)^{2}}\end{array}$ & $<5 \mu \mathrm{m}$ & $<7 \mu \mathrm{m}$ & $\begin{array}{c}E=13.6 \mathrm{GeV} \\
Q_{0}=3 \mathrm{~T} \\
\beta=10 \mathrm{~m} \\
\varepsilon_{Q}=0.35 \\
\text { other values as } \\
\text { above }\end{array}$ \\
\hline Undulator & & $\sim 10 \mu \mathrm{m}$ & $\sim 10 \mu \mathrm{m}$ & $\begin{array}{l}\text { Field error dependent, } \\
\text { vertical only }\end{array}$ \\
\hline
\end{tabular}

${ }^{\mathrm{a} E r r o r s}$ associated with mechanical reproducibility.

optics, especially when differential measurements are performed against a standard undulator [26].

Table V summarizes the estimated alignment errors for the LCLS undulator components. Additional error estimates for OTR and quadrupole positioning are also provided. Reference [24] gives further details.

\section{FURTHER DISCUSSIONS}

In this section, we will discuss several practical issues related to the application of x-ray beam alignment and further enhancements of this technique.

\section{A. Relation with electron-beam-based alignment}

Let us consider a magnetic lattice consisting of only quadrupole magnets. When all centers of the magnets are aligned on a straight line and the electron beam travels along the line, the electron trajectory is independent of beam momentum or magnetic field strength. Otherwise, when scanning the electron energy or the strengths of the magnet fields, the trajectory will change, giving telltale signs of the misalignment and clues for correction. By correctly interpreting the trajectory changes during energy scans or field scans, the electron-beam-based alignment technique has matured in the past decade, and can attain micron-level accuracy under ideal conditions [11,12]. However, the EBBA technique is sensitive to the perturba- tion of a dipole field. Even the Earth's field may adversely affect the outcome. Other engineering limitations may also come into play. For example, a slight asymmetry of a magnet's core may generate an unintentional dipole field within and move the magnetic center during magnet field scans. Similarly, an asymmetry in the rf accelerating structure may generate energy-dependent transverse deflections of the beam during energy scans. Finally, since no fixed monument is used to define the EBA, it is not predetermined to what line the final convergence will lead.

The x-ray beam-based alignment (XBBA) can enhance the EBBA in two aspects:

(i) Using the fiducialization/alignment technique described in the previous section, all magnetic quadrupoles and undulators can be positioned within several micrometers from the XBA, providing the EBBA with an excellent starting configuration.

(ii) By forcing the EBA to coincide with the XBA, we obtain a high reproducibility inherently lacking in the EBBA. This can be achieved in several ways: (1) Start from a configuration near the XBA and impose appropriate constraints during the entire EBBA process to prevent the EBA from wandering too far from the XBA. (2) Beam position measurements using fluorescent screens along the accelerator can be used to quantify differences between the two axes after EBBA convergence. If a systematic de- 


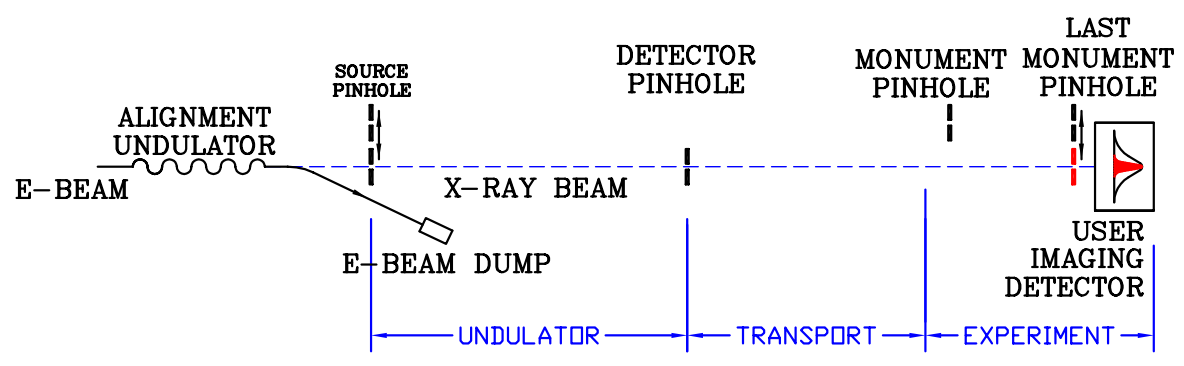

FIG. 9. (Color) Extension of XBA into transport and user experimental area.

viation is found, the entire beam line can be rotated to force the EBA to coincide with the XBA. When this is accomplished, the user experiments can enjoy reproducible beam position and direction from one run to the next.

When the undulators' first field integrals are too high, the EBBA in the undulator hall will not be able to converge to a straight line. The XBA may be used as a quality control tool to monitor the final EBA to prevent this from happening. In the rare case when the EBBA does not converge, the XBA provides a fallback position.

\section{B. Extension of XBA to the user area}

From Sec. II, we can see that the rms alignment error increases as the square root of the distance between the source and detector pinholes. Hence the main beam line should be kept as short as possible, containing only those components requiring critical alignment plus an adequate working distance upstream and downstream of the main undulator section. In order to align the x-ray transport line and the user beam line components, we need to extend the alignment monuments for the XBA beyond the detector pinhole. The following procedure may be used for this purpose (Fig. 9):

(i) Set up the alignment undulator (Sec. II). Insert the source and detector pinholes and remove the imaging detector behind it.

(ii) Set up an imaging detector/PSD at the end of the $\mathrm{x}$ ray transport line and user experiment, and record the center of the $\mathrm{x}$-ray beam spot.

(iii) Install an x-ray pinhole just upstream of the detector. Insert it in the beam and move it until the centroid of the beam spot coincides with the recorded center in the last step.

The XBA is now extended up to the last $\mathrm{x}$-ray pinhole in the transport line. Other pinholes and monuments can be aligned using the procedure described in Sec. II, with the last pinhole serving as the new detector pinhole. Note that the monuments established with this procedure have slightly poorer accuracy than the pinholes inside the accelerator. In most cases however, it is not a major problem since the transport line and user beam line usually require less stringent alignment in the machine coordinate system.
We note that the extension of the XBA into the $\mathrm{x}$-ray beam line is especially important for $\mathrm{X}$-ray diagnostics of the undulator and the FEL, since the x-ray alignment sets up an absolute angle reference for the undulator radiation pattern, enabling diagnostics of the electron trajectory within the magnetic structure of the undulator.

\section{Enhancement of positioning accuracy and photon efficiency with zone plate targets}

From Sec. II, we note that the optimum target pinhole size increases as the square root of the beam line length $S$ and $\mathrm{x}$-ray wavelength $\lambda$. It follows that the area of the pinhole increases linearly with the beam line length, and its acceptance solid angle for $\mathrm{x}$-ray photons remains unchanged. Hence the photon statistic is independent of the beam line length. However, the enlargement of the target pinhole in longer accelerators or lower-energy machines, resulting in longer $\mathrm{x}$-ray wavelength, does increase the alignment error.

In these cases, one may consider using Fresnel zone plates to improve the positioning accuracy. The x-ray beam spot size at the detector plane can be calculated using standard Fresnel diffraction formulas [26,27]. The result is

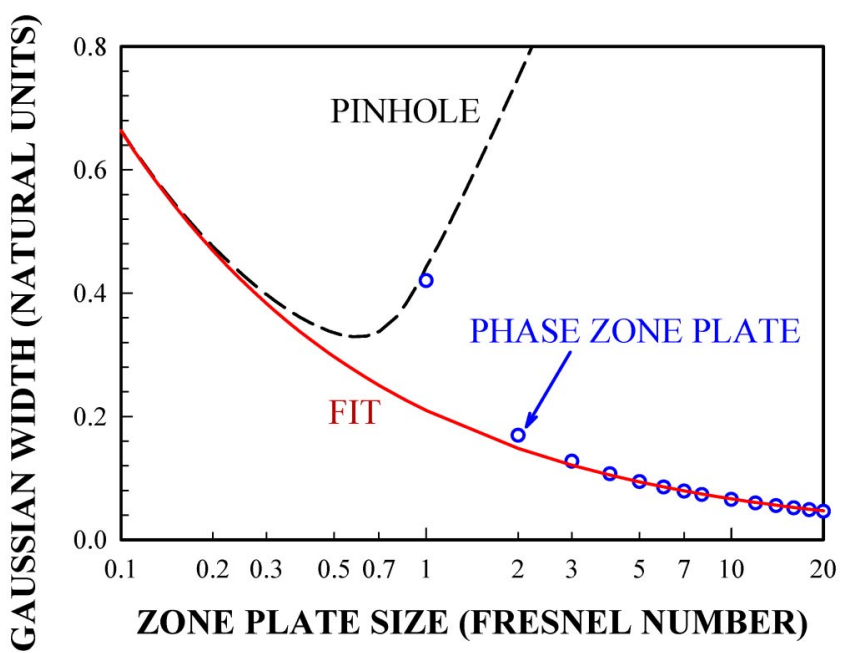

FIG. 10. (Color) Calculated Gaussian width of image profiles of a zone plate camera: (circle) phase zone plates, (dashed line) pinhole, and (solid line) fit for high Fresnel number cameras. 
shown in Fig. 10. A phase zone plate with even a handful of zones $(\geq 4)$ can improve the resolution over the optimal pinhole by a factor of 3 or more. At high Fresnel numbers, the Gaussian peak width of the diffraction profile at the focal plane $\sigma_{2 \text {,reso }}$ is given by

$$
\frac{\sigma_{2, \text { reso }}^{2}}{(M+1)^{2} \lambda f}=\frac{0.044}{F} .
$$

Hence the resolution improvement is proportional to the square root of the number of the Fresnel zones.

$\mathrm{X}$-ray zone plates can also be used to improve the photon collection efficiency since the area of the zone plates, and hence the photon counts, are proportional to the number of zones. This is especially useful for a long accelerator such as the ILC.

\section{Planning and designing the XBA monuments}

The source and detector pinholes are the master alignment monuments when inserted in the $x$-ray beam line. They define the positions of the entire XBA, EBA, and hence all accelerator components between the two monuments. Their positions need to be carefully chosen, their supports need to be carefully designed, and their insertion mechanisms need to be accurately engineered allowing greatest reproducibility and longest uninterrupted service.

In the coordinate system defined by these two monuments, the position of the $i$ th accelerator component can be written as $\left(x_{i}, y_{i}, z_{i}\right), i=1, \ldots, M$. A straight-line average beam axis can be established by fitting the positions of the accelerator components

$$
X_{i}=\bar{X}_{S}+\frac{z_{i}}{S}\left(\bar{X}_{D}-\bar{X}_{S}\right)
$$

where $\bar{X}_{S}$ and $\bar{X}_{D}$ are fitting parameters. They are determined by the equations $\sum_{i=1}^{M} \Delta x_{i}=0$ and $\sum_{i=1}^{M} \Delta x_{i} z_{i}=0$, where $\Delta x_{i} \equiv x_{i}-X_{i}$ is the component offset from the fitted line. The distance between the source pinhole and the average beam axis is given by $X_{S}=-\bar{X}_{S}$ and that of the detector pinhole by $X_{D}=-\bar{X}_{D}$. By convention, the deviation from the average beam axis $\Delta x_{i}$ can be taken as the alignment error of the component. Its rms value follows the "ATL law," showing that the ground motion is diffusionlike [28]

$$
\left\langle\Delta x^{2}\right\rangle=A \cdot t \cdot S
$$

with the coefficient $A$ in the range of $1 \sim 100 \mu \mathrm{m}^{2}$ per day per $100 \mathrm{~m}$, depending on local geological conditions, $t$ representing the time interval between measurements, and $S$ the distance between observation points. Averaging the expression, $x_{i}^{2}=\left(\Delta x_{i}+X_{i}\right)^{2}$, we obtain

$$
\begin{aligned}
\left\langle x^{2}\right\rangle & =\left\langle\Delta x^{2}\right\rangle+\left\langle X^{2}\right\rangle \\
& =\frac{1}{M} \sum_{i=1}^{M} \Delta x_{i}^{2}+\frac{1}{6}\left[X_{D}^{2}+\left(X_{S}+X_{D}\right)^{2}+X_{S}^{2}\right],
\end{aligned}
$$

where we have used Eq. (24) to estimate $\left\langle X^{2}\right\rangle$. This expression confirms our intuition that the position accuracy of the pinhole monuments carries more weight than any alignment target. Therefore, several issues need to be carefully considered to position these monuments.

(i) Reproducible insertion mechanism: From Eq. (26), we can see that the uncertainty of the monument positions can easily contribute half of the rms error, and a large rms alignment error can be created instantly by just exercising a poorly designed insertion mechanism. Fortunately, much progress has been made in the past decades in the field of precision machining technology with micron and submicron reproducibility [29]. Applying kinematic mounting technology, the semiconductor industry developed submicron wafer positioning systems in a clean room. Therefore, insertion mechanisms with several microns reproducibility should be within reach for a temperature-controlled accelerator tunnel.

(ii) Stable support stand: Strong support stands are required so that the amplitude of the vibrationinduced monument motion is well within the alignment tolerance.

In addition to these technical considerations, a number of design issues depend strongly on the operational philosophy of the accelerator:

(i) Should the positions of the alignment monuments be adjustable, or even remotely adjustable?

(ii) For thermal stability of the design monument, should one use low-thermal expansion materials such as INVAR, or attempt to tightly control the support temperature using well-regulated coolant flowing through the stand? On the other hand, one has to ask if it would be advantageous to make the support from the same material as other accelerator components, such as support girders, and allow the monuments to drift up and down with the accelerator.

(iii) For the installation site, one has to evaluate if these monuments should be anchored to the bedrock, or if they should float with the tunnel floor that supports all the other accelerator components.

The answers to these questions depend largely on the manner of applying corrections after component position errors are measured. Will one move the components to a fixed XBA, or will one move the XBA to the average line first before moving other accelerator components? In an XFEL user facility where a stable output beam position is required, a fixed XBA is highly desirable. According to Eq. (26), the rms error and the needed component position 
correction in this operating mode will be about twice as large as using the floating average beam axis.

\section{E. Challenges in mechanical design}

In order to implement the technique proposed in this work and to obtain micron accuracy, we are facing significant challenges in mechanical engineering:

(i) In order to transfer the accurate position information from the pinholes to the attached component(s), the target insertion mechanism needs to have comparably high reproducibility. State-ofthe-art kinematic mounts with submicron accuracy at high load $(>100 \mathrm{~N})$ need to be employed [29]. In addition, the repeatability of the retractable pinholes is a major source of error for transferring the position information to an outside fiducial. When such fiducials are needed, ultrahigh-precision machining and motion systems will be required.

(ii) To use fiducialized screens and wire scanners as beam position monitors, not only should their motion resolution be finer than the required accuracy, but their motion reproducibility should also be comparable. Nanopositioning systems are now commercially available and should be utilized.

(iii) To maintain micrometer positioning accuracy for accelerator components, the support and mover system of the component(s) needs to be stable, and loads need to be carefully distributed. For example, it is highly desirable to separate supports of quadrupole magnets and BPMs, which have very high stability requirements, from those of heavy undulators, which have less stringent stability requirements and need to be rolled in and out at the LCLS or opened and closed in other facilities.

\section{F. Real-time component position monitor}

Even when the mechanical design meets all the challenges, environmental changes may still be beyond human control. A temperature change of $1{ }^{\circ} \mathrm{C}$ can easily move the target on a 1-m-high support stand by $10 \mu \mathrm{m}$ or more. The temperature variation of the floor, the wall, and the support stand will easily move accelerator components on a micrometer scale. Even if we have the enclosure temperature under rigorous control, the daily tidal motion of the Earth's surface will move the ground up and down by up to $\pm 30 \mathrm{~cm}$ in a 12-hour period, resulting in some twisting and bending of the floor. While the magnitude of the twisting and bending depends on local geological conditions, a reproducible alignment literally depends on the phase of the moon. In addition to these periodic movements, the Earth's inelastic crust releases its internal stress by a natural diffuse settlement process. There are many sources of the stress that range from local temperature effects to large-scale continental motions. Therefore, any well-aligned configuration will drift out of alignment slowly, as described by the "ATL law."

In accelerators where component movements are not acceptable after applying all available passive mitigation

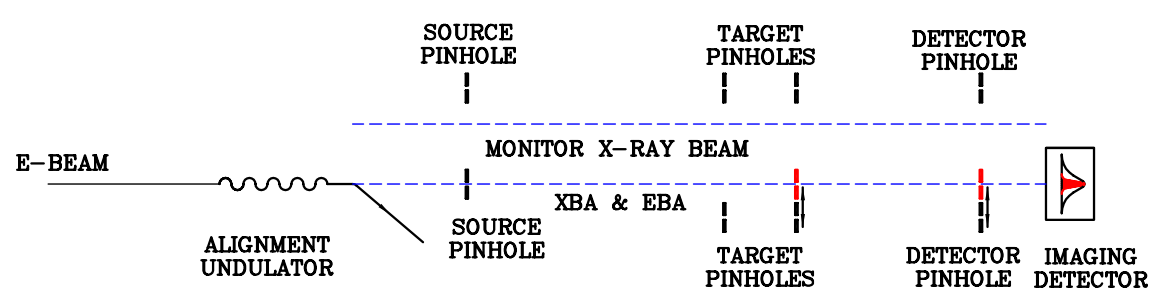

(a) Align accelerator (on-axis)

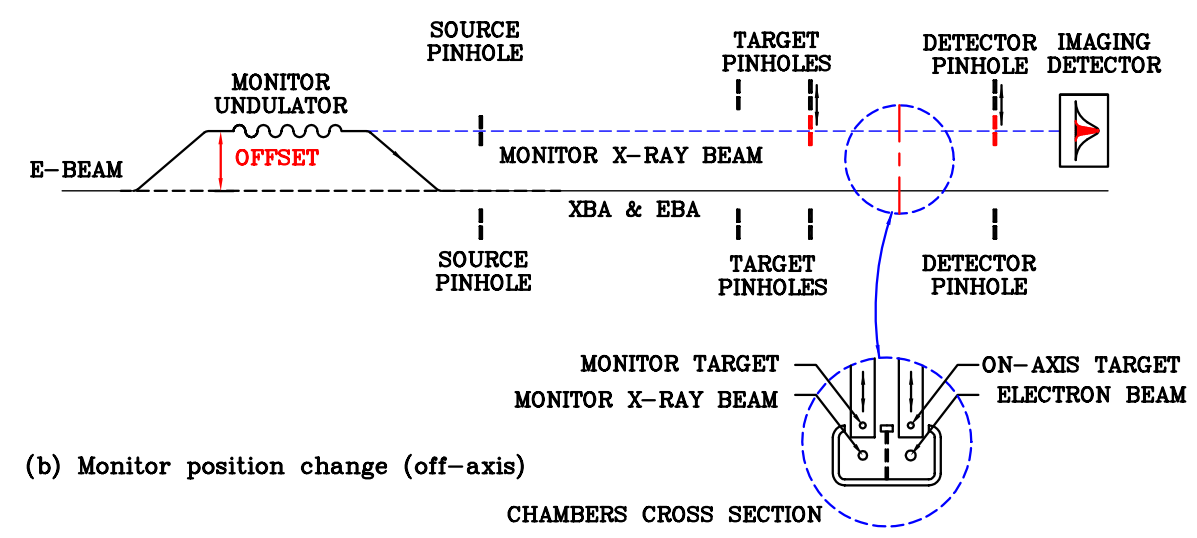

FIG. 11. (Color) Real-time position monitoring system: (a) Alignment with on-axis trajectory and targets. (b) Observe position changes with off-axis monitoring X-ray beam line. The inset shows two independently retractable pinhole targets, one used for XBA and the other for monitoring position changes. In an x-ray FEL, the monitoring beam line has its own vacuum chamber to reduce the radiation background. 
approaches, a regular observe-and-correct procedure must be implemented. The target pinholes attached to critical components can be inserted and their positions measured at regular time intervals. In the case of LCLS, a period of several hours or days may suffice. Over time this approach will provide valuable information about long-term ground settlement.

In case higher frequency observations and corrections are required, a real-time position monitoring and feedback system must be considered. Figure 11 shows a possible implementation using an off-axis x-ray alignment beam line. The electron beam is steered through a chicane or a two-stage trajectory offset before entering the main undulator hall. The offset of the two beams are adjustable from zero to a maximum of several millimeters. Each critical component will have two independently retractable pinhole targets, one on axis and the other off axis, in the monitor beam line. The monitor beam line is parallel to the EBA and has its own independently operated source and detector pinholes, as well as its own detector system.

During the on-axis alignment procedure [Fig. 11(a)], the trajectory offset is set to zero and the on-axis target is used to position the critical component(s) as described in the previous sections. After the alignment is completed, we set the offset electron trajectory to be collinear with the monitor beam line [Fig. 11(b)]. The same undulator now produces x-ray beam along the parallel monitor beam axis, which can be used to fiducialize the off-axis monitor pinhole target without moving the component. From now on, any change in the position of the off-axis target pinhole will shift the pinhole camera image on the detector, providing real-time information on the component position change at a resolution of micrometers. The information will allow position adjustments to be made while the accelerator is running. Since only one target is inserted at any given time, the wakefield effect of the target may be negligible. However, in order to use such a monitorfeedback system for position control of an x-ray FEL, a larger separation of the two beam lines, even separate beam pipes, may be required to avoid contamination of the position monitor signal by the FEL beam.

X-ray Fresnel lenses are ideally suited for real-time component position monitoring in long accelerators. Their small $\mathrm{x}$-ray spots allow micron-level resolution in component position. Their high acceptance angles make good use of the x-ray flux and allows faster measurement. Finally, since the monitoring beam line measures only relative changes of a component position, the difficulty associated with fiducializing large zone plates becomes unimportant for the application.

\section{SUMMARY AND CONCLUSION}

We proposed a new alignment technique using an undulator x-ray beam produced by high-energy electrons. The proposal contains two major technical components:
First, two solidly built x-ray pinholes, source and detector, are used to define a highly stable and reproducible $\mathrm{X}$-ray beam axis (XBA). Second, the XBA is chosen to be collinear with the electron-beam axis in order to minimize the transfer errors to accelerator components and maximize the coincidence between $\mathrm{x}$-ray beam and e-beam.

We showed that a measurement accuracy of $1-3 \mu \mathrm{m}$ can be achieved for target pinholes in the 200-m-long LCLS undulator hall. The accuracy can be further enhanced with $\mathrm{x}$-ray zone plates, if necessary. We described procedures to transfer this position information in situ to quadrupoles, diagnostic components, undulators, and user beam lines, at an overall accuracy of better than $10 \mu \mathrm{m}$ in the case of the LCLS undulator.

This $\mathrm{x}$-ray alignment technique also enhances the electron-beam-based alignment (EBBA) process in three aspects: First, it provides a good starting configuration for the EBBA. Second, it provides fixed anchors for the electron-beam axis (EBA) for reproducible trajectories from run to run. Last but not least, it facilitates the extension of the coordinate axes into the user area at high accuracy, enabling far-field x-ray diagnostics to perform angular distribution measurements with absolute angle reference.

Finally, we addressed the possibility to utilize this technique for a real-time position monitor system of critical beam components of an FEL or a long accelerator. It will enable active position control to micrometer levels.

Although we used the LCLS parameters for this paper, other FELs currently in the design stage could benefit by integrating such a system from the beginning. The proposed alignment system promises superior alignment accuracy over long distances and may therefore be a viable approach for aligning beam components of the International Linear Collider (ILC) currently under consideration.

\section{ACKNOWLEDGMENTS}

We would like to thank Paul Emma and Heinz-Dieter Nuhn for stimulating discussions on electron-beam-based alignment techniques, and Steve Milton for his encouragement for this work. This work is supported by the U.S. Department of Energy, Office of Basic Energy Sciences, Office of Science, under Contracts No. W-31-109-ENG-38 and No. DE-AC03-76SF00515.

[1] J. Arthur, P. Anfinrud, P. Audebert, K. Bane, I. Ben-Zvi, V. Bharadwaj, R. Bionta, P. Bolton, M. Borland, P. H. Bucksbaum, R. C. Cauble, J. Clendenin, M. Cornacchia, G. Decker, P. Den Hartog, S. Dierker, D. Dowell, D. Dungan, P. Emma, I. Evans, G. Faigel, R. Falcone, W. M. Fawley, M. Ferrario, A. S. Fisher, R. R. Freeman, J. Frisch, J. Galayda, J.-C. Gauthier, S. Gierman, E. Gluskin, W. Graves, J. Hajdu, J. Hastings, K. Hodgson, 
Z. Huang, R. Humphry, P. Ilinski, D. Imre, C. Jacobsen, C.-C. Kao, K. R. Kase, K.-J. Kim, R. Kirby, J. Kirz, L. Klaisner, P. Krejcik, K. Kulander, O. L. Landen, R. W. Lee, C. Lewis, C. Limborg, E. I. Lindau, A. Lumpkin, G. Materlik, S. Mao, J. Miao, S. Mochrie, E. Moog, S. Milton, G. Mulhollan, K. Nelson, W. R. Nelson, R. Neutze, A. Ng, D. Nguyen, H.-D. Nuhn, D. T. Palmer, J. M. Paterson, C. Pellegrini, S. Reiche, M. Renner, D. Riley, C. V. Robinson, S.H. Rokni, S. J. Rose, J. Rosenzweig, R. Ruland, G. Ruocco, D. Saenz, S. Sasaki, D. Sayre, J. Schmerge, D. Schneider, C. Schroeder, L. Serafini, F. Sette, S. Sinha, D. van der Spoel, B. Stephenson, G. Stupakov, M. Sutton, A. Szöke, R. Tatchyn, A. Toor, E. Trakhtenberg, I. Vasserman, N. Vinokurov, X.J. Wang, D. Waltz, J.S. Wark, E. Weckert, H. Winick, M. Woodley, A. Wootton, M. Wulff, M. Xie, R. Yotam, L. Young, and A. Zewail, Report No. SLAC-R-593, http://www.slac.stanford.edu/ pubs/slacreports/slac-r-593.html

[2] J. Pflüger, in Proceedings of the Workshop on Undulator Systems for X-FELs, WUS2005, DESY Hamburg, Germany, 2005, http://adweb.desy.de/ faatz/wus2005/ program.html

[3] T. Shintake, in Proceedings of the Third Asia Particle Accelerator Conference, Gyeongju, Korea, March 2004 (to be published at http://www.jacow.org).

[4] Y. Kim, J.S. Oh, M. H. Cho, I. S. Ko, W. Namkung, D. Son, and Y. Kim, in Proceedings of the Third Asia Particle Accelerator Conference, Gyeongju, Korea, March 2004 (Ref. [3]).

[5] J. Prenting, M. Schlösser, A. Herty, J. Green, G. Grzelak, A. Mitra, and A. Reichold, in Proceedings of the XXII International Linear Accelerator Conference, Lübeck, Germany, 2004, pp. 690-692, http://www.jacow.org

[6] P. Tenenbaum, in Proceedings of the ILC Meeting at KEK, Tsukuba, Japan, 2004, http://lcdev.kek.jp/ILCWS/Talks/ 13wg1-4-linacemit_KEK_PT.pdf

[7] W. Schwarz, Vermessungsverfahren im Maschinen- und Anlagenbau (Konrad Wittwer, Stuttgart, Germany, 1995).

[8] W. B. Herrmannsfeldt, M. J. Lee, J. J. Spranza, and K. R. Trigger, Appl. Opt. 7, 995 (1968).

[9] L. V. Griffith, R. F. Schenz, and G. E. Sommergren, Rev. Sci. Instrum. 61, 2138 (1990).

[10] I. Feier, H. Friedsam, and M. Penicka, in Proceedings of the International Workshop on Accelerator Alignment, Argonne National Laboratory, 1997, http://wwwgroup.slac.stanford.edu/met/IWAA/IWAAHome.html

[11] P. Tenenbaum and T. O. Raubenheimer, Phys. Rev. ST Accel. Beams 3, 052801 (2000).

[12] C. Adolphsen, T. L. Lavine, W. B. Atwood, T. M. Himel, M. J. Lee, T. S. Mattison, R. Pittman, J. T. Seeman, S. H.
Williams, and G.H. Trilling, in Proceedings of the Particle Accelerator Conference, Chicago, IL, 1989 (IEEE, Piscataway, NJ, 1989), pp. 977-9979, http:// www.jacow.org.

[13] A.E. Siegman, Lasers (University Science Books, Mill Valley, CA, 1986).

[14] T. Shintake, H. Kitamura, and T. Ishikawa, in Synchrotron radiation instrumentation: Eighth International Conference on Synchrotron Radiation Instrumentation, AIP Conf. Proc. No. 705 (AIP, New York, 2004), p. 117.

[15] M. Borland, "A Method for Calculating Emittance from Undulator Images," SSRL ACD-NOTES 60, 1989.

[16] B.X. Yang and A. Lumpkin, in Proceedings of the 1999 Particle Accelerator Conference, New York, 1999 (IEEE, Piscataway, NJ, 1995), pp. 2161-2163, http://www. jacow.org.

[17] B. X. Yang, in Proceedings of BIW'02, Upton, New York, 2002, AIP Conf. Proc. No. 648 (AIP, New York, 2002), pp. 59-78.

[18] P. Elleaume, C. Fortgang, C. Penel, and E. Tarazona, J. Synchrotron Radiat. 2, 209 (1995).

[19] Z. Cai, B. Lai, W. Yun, E. Gluskin, D. Legnini, P. Illinski, and G. Srajer, Rev. Sci. Instrum. 67, 3368 (1996).

[20] H. Nuhn, "LCLS Undulator Requirements," LCLS Specification 1.4-001.

[21] J. Kirz et al., X-ray Data Booklet (Lawrence Berkeley Lab, Berkeley, CA, 1986), and references cited therein.

[22] M. Sanchez del Rio and R. J. Dejus, in Proc. SPIE Int. Soc. Opt. Eng. 3448, 340 (1998).

[23] K. J. Kim, in Physics of Particle Accelerators: Fermilab Summer School 1987, Cornell Summer School 1988, edited by Melvin Month and Margaret Dienes, AIP Conf. Proc. No. 184 (AIP, New York, 1989), p. 565.

[24] B. Yang and H. Friedsam, ANL Report No. ANL/ APS/LS-310, 2005, http://www.aps.anl.gov/Facility/ Technical_Publications/lsnotes/.

[25] T. O. Raubenheimer and D. Schulte, in Proceedings of the 1999 Particle Accelerator Conference, New York, edited by A. Luccio and W. MacKay (IEEE, Piscataway, NJ, 1999), pp. 3441-3443, http://www.jacow.org.

[26] B.X. Yang, in Proceedings of the 2005 Particle Accelerator Conference, Knoxville, TN, 2005 (IEEE, Piscataway, NJ, 2005), pp. 2342-2344, http://www. jacow.org.

[27] M. Born and E. Wolf, Principles of Optics: Electromagnetic Theory of Propagation, Interference and Diffraction of Light (Cambridge University Press, Cambridge, UK, 1997).

[28] V. Shiltsev, Report No. FERMILAB-FN-0717, 2002.

[29] A. Slocum, Precision Engineering 14, 67 (1992). 